${ }^{6}$ Uchida, T., Pappenheimer, A. M. jun., and Harper, A. A., Science, 175, 901 (1972).

7 Waud, D. W., Pharmacol. Rev., 20, 49 (1968).

${ }^{8}$ Schild, H. O., Pharmacol. Rev., 9, 242 (1957).

${ }^{9}$ Gaddum, J. H., Pharmacol. Rev., 9, 211 (1957).

${ }^{10}$ Goor, R. S., Nature, 217, 1051 (1968).

11 Strauss, N., and Hendee, E. D., J. Exp. Med., 109, 144 (1959).

${ }_{12}$ Moehring, T. J., Moehring, J. M., Kuchler, R. J., and Solotorovsky, M., J. Exp. Med., 126, 407 (1967).

\section{Senescence of Human Cells in Culture}

DR L. K. BLEYMAN ${ }^{1}$ need not be concerned that our experiments on clonal senescence of human cells in culture are being carried out in ignorance of earlier studies of such ageing in protozoa. Indeed the paper he cites ${ }^{2}$ refers to one important study carried out with protozoa. The experiments with human cells are derived directly from earlier work with simple eukaryotic organisms such as fungi. When the first results were being prepared for publication ${ }^{3}$, reference was made to related studies with ciliated protozoa and a copy was sent to Professor T. M. Sonneborn for comment. In subsequent correspondence with him it became clear that it would not be possible (in a paper limited in length by the editor of Nature) to do justice to the many important observations on the complex interaction between nuclear and cytoplasmic degenerative changes during ageing in these organisms. It would be most valuable if $\mathrm{Dr}$ Bleyman could now be persuaded to publish a review of these experiments in the light of subsequent biochemical studies on the mechanism of clonal senescence in other organisms.

\section{ROBIN HOLLIDAY}

MRC National Institute for Medical Research,

\section{Mill Hill,}

London NW7 1AA

${ }^{1}$ Bleyman, L. K., Nature, 240, 152 (1972).

2 Holliday, R., and Tarrant, G., Nature, 238, 26 (1972).

3 Holliday, R., Nature, 221, 1224 (1969).

\section{Circadian Variation of the Lethality of Methadone}

Lenox and Frazier ${ }^{1}$ have reported the discovery of a circadian variation of methadone tolerance in rats. Their claim hinges on an observed survival of nine out of twenty-six rats $(34.6 \%)$ injected at $0500 \mathrm{~h}$ compared to an average survival of only $17.3 \%$ for similar groups injected at five other times of day. But their use of the $\chi^{2}$ test is incorrect and the experimental results do not support the hypothesis that the circadian cycle influences the tolerance to methadone.

To calculate the significance of the observed variation of survival percentage with time of injection it is necessary to recognize that survival statistics in experiments with small groups are neither Gaussian nor Poisson, but are binomial ${ }^{2}$. Whenever an experiment with a group of size $n$ has only two possible outcomes (such as survival and death) with probabilities $p$ and $q(q=1-p)$ the probability of $x$ survivals is given by

$$
P(x)=n !(x !(n-x) !)^{-1} p^{x} q^{n-x}
$$

The best estimate of $p$, under the null hypothesis that the survival rate is independent of time of injection, is defined by the observed survival of 31 animals out of the 153 used in the programme of six experiments. Therefore $p=0.203$ and $q=0.797$. The binomial function based on these values for $p$ and $q$, with $n=26$, as for the group at $0500 \mathrm{~h}$, is shown in Fig. 1 . The sum of the ordinates from $x=0$ to $x=8$ is equal to 0.936

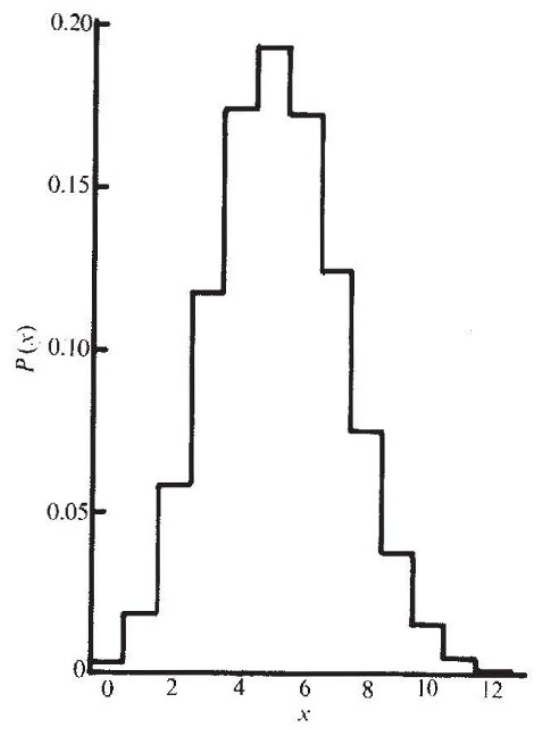

Fig. 1 The binomial function for $n=26$ and $p=0.203$.

and therefore the likelihood of 9 or more survivals is $6.4 \%$ in this one group. But there were five other groups, any one of which could have produced an interesting result. The probability of obtaining eight or fewer survivals in all six groups is equal to the product of the six separate probabilities. There are only two distinct probabilities to consider: 0.936 for $n=26$ in three experiments and 0.950 for $n=25$ in the other three. The overall probability that $x<9$ is therefore $0.936^{3} \times 0.950^{3}=0.703$. It follows that there will be a $29.7 \%$ chance that $x>8$ in at least one of the six groups, and the null hypothesis is compatible with the observations.

Statistical interpretation of the results of this experiment is complicated by the fact that previous work cited by Lenox and Frazier had suggested that survival would be greatest at $0900 \mathrm{~h}$, only one time interval away from the $0500 \mathrm{~h}$ group. If the work is repeated it will require only eight survivals at $0500 \mathrm{~h}$ or $0900 \mathrm{~h}$ to rule out the null hypothesis.

Unless there are compelling biological reasons for using an $\mathrm{LD}_{80}$ dose of methadone the sensitivity of the experiment can be doubled by employing the drug at the $\mathrm{LD}_{50}$ level.

Edward Argyle

\section{Dominion Radio Astrophysical Observatory, Penticton, $B C$}

Received November 3, 1972.

${ }^{1}$ Lenox, R. H., and Frazier, T. W., Nature, 239, 397 (1972).

${ }^{2}$ Hoel, P. G., Introduction to Mathematical Statistics, 38 (Wiley, New York, 1947).

\section{Attraction of Spodoptera littoralis Larvae to Colours}

DURING a research programme on phagostimulants ${ }^{1-8}$ for the larva of the Egyptian cotton leafworm, Spodoptera littoralis Boisd., it was found that their incorporation into poison baits did not sufficiently improve the latter's attractivity. Further stimuli, for example optical ones, seemed to be needed. We therefore determined the influence of edible colours, similar to those used in studying boll weevil attraction ${ }^{4}$, on the feeding rate of $S$. littoralis. A range of commercial food colours of the 'Edicol Supra' and 'Edilake' ranges (Imperial Chemical Industries, England), now produced under the label of 'Certicols' and 'Certolakes', respectively (Williams (Hounslow) Ltd), were tested. The 'Edilake (Certolake)' colours are aluminium lakes containing a high proportion of alumina as extender of the corresponding 'Edicol Supra (Certicol)' dyes, which are sodium 